\title{
Form factors from lattice QCD
}

\section{Dru B. Renner*}

Thomas Jefferson National Accelerator Facility (JLab)

E-mail: druajlab.org

Precision computation of hadronic physics with lattice QCD is becoming feasible. The last decade has seen percent-level calculations of many simple properties of mesons, and the last few years have seen calculations of baryon masses, including the nucleon mass, accurate to a few percent. As computational power increases and algorithms advance, the precise calculation of a variety of more demanding hadronic properties will become realistic. With this in mind, I discuss the current lattice QCD calculations of generalized parton distributions with an emphasis on the prospects for well-controlled calculations for these observables as well. I will do this by way of several examples: the pion and nucleon form factors and moments of the nucleon parton and generalizedparton distributions.

Sixth International Conference on Quarks and Nuclear Physics

April 16-20, 2012

Ecole Polytechnique, Palaiseau, Paris

${ }^{*}$ Speaker. 


\section{Introduction}

Over the last decade, lattice QCD has proven itself capable of percent-level calculations. This is a milestone that establishes lattice field theory as a powerful tool for performing reliable and quantitative computations of nonperturbative QCD phenomena. However, it is important to understand that the computational demands required for such calculations depend strongly on the quantities of interest. Consequently, the most impressive lattice calculations to date have been limited to arguably the simplest QCD observables, mostly meson properties. Recently, there has been progress on the determination of the baryon spectrum. In particular, the nucleon mass can now be calculated nonperturbatively from QCD at the few-percent level, raising the prospects for calculations of more challenging observables that have long been sought from lattice QCD.

Even with the successful computation of the nucleon mass, the calculation of nucleon form factors remains a challenge for lattice QCD. Significant progress has been made in the last few years. In particular, recent computations with nearly physical pion masses represent a major breakthrough for lattice QCD and have been essential to the successful determination of the nucleon mass, which is clearly a necessary first step. However, there are additional sources of uncertainty that occur for calculations of nucleon matrix elements. With nearly physical pion masses becoming more common, the focus is turning toward fully controlling all uncertainties relevant to the computation of matrix elements in order to perform calculations of form factors that can be quantitatively compared to experimental measurements.

In these proceedings, I start by briefly reviewing several examples of high-precision lattice calculations and the recent precision determinations of the masses of baryons, in particular the nucleon. I then turn to the pion form factor as a simple example of the more challenging quantities that our community is interested in. The calculation of this observable provides a glimpse of the progress that we can hopefully expect for nucleon form factors in the years to come. However, nucleon calculations are still an open issue, so I finish by looking at just a few representative examples of the generalized parton distributions of the nucleon.

\section{High-precision lattice QCD calculations}

There is now a small but growing list of quantities that can be calculated using lattice QCD with percent-level accuracy. Figure 1 shows several. In order to understand if and when such precision will be brought to bear on more challenging observables, it is important to note that the earliest calculations in figure 1 were only performed within the last decade. Percent-level precision has been reached for additional quantities since then, but almost all of these are related to simple properties of stable mesons. Despite these limitations, the results in figure 1 represent a significant accomplishment of the lattice community and demonstrate that high-precision calculations are possible using lattice QCD.

\section{Precision lattice QCD computation of baryon masses}

There is a notable absence of baryon properties in figure 1. For a variety of reasons, calculations involving baryons require significantly more computational resources that those for mesons. 


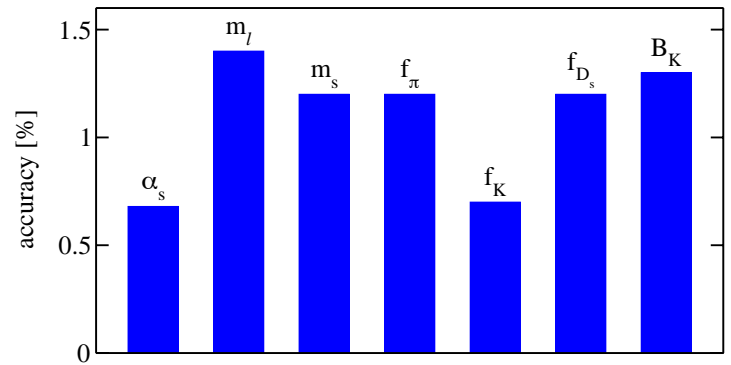

Figure 1: Examples of high-precision lattice QCD calculations. Several observables have been calculated with a total precision of one percent or better, illustrating that lattice QCD is capable of highprecision computations. The quantities shown here are averages of several lattice calculations $[1,2]$.

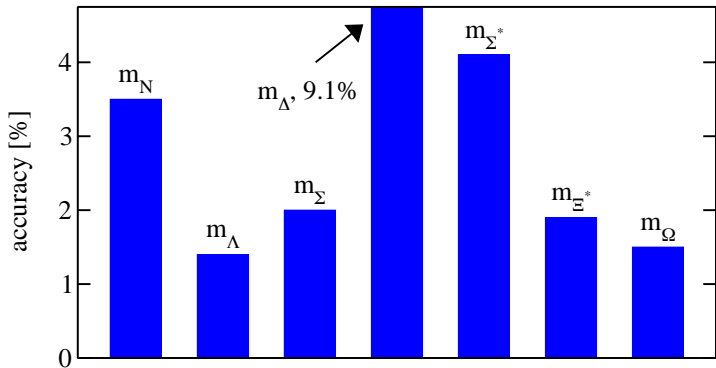

Figure 2: An example precision computation of baryon masses. Lattice computations involving baryons are more challenging than mesons and have only recently reached the few-percent level. The masses from BMW [3] are shown, but PACS-CS [4] and ETMC [5] find similar results.

In particular, nucleon properties appear to have a more significant dependence on the pion masses used for their determinations. This represents a computational threshold that afflicts the calculation of nucleon observables more than others. Nonetheless, calculations completed in just the last few years were the first to demonstrate some quantitative control over the ground-state baryon masses shown in figure 2. The determination of the nucleon mass $m_{N}$ at the few-percent level is an important advance forward for the lattice community. It raises the prospects that the more challenging task of computing nucleon matrix elements will be within the reach of lattice QCD. However, we should bear in mind that the calculation of $m_{N}$ has only been possible in the last few years. This suggests that nucleon matrix elements may very well require yet more time.

\section{Lattice QCD calculation of the pion form factor}

The pion electromagnetic form factor has recently started to yield to quantitatively controlled lattice calculations. It serves as an example of what to expect for nucleon form factors in the years to come. Figure 3 shows a summary plot of lattice QCD calculations of the charge radius of the pion $\left\langle r^{2}\right\rangle_{\pi}$, defined by

$$
\left\langle\pi, p\left|J_{\mu}\right| \pi, k\right\rangle=\left(p_{\mu}+k_{\mu}\right) F\left(Q^{2}\right) \quad Q^{2}=-(p-k)^{2} \quad\left\langle r^{2}\right\rangle_{\pi}=-\left.6 \frac{d F\left(Q^{2}\right)}{d Q^{2}}\right|_{Q^{2}=0},
$$

where $J_{\mu}=\sum_{f} Q_{f} J_{\mu}^{f}, J_{\mu}^{f}=\bar{q}_{f} \gamma_{\mu} q_{f}$ and $Q_{f}$ is the electric charge for each quark flavor $f$. There appears to be no agreement amongst the lattice calculations, some of which do and some do not agree with the experimentally measured value of $\left\langle r^{2}\right\rangle_{\pi}$. As I will argue shortly, this plot is misleading, but I have chosen to examine it as an example of how lattice QCD calculations progress.

To understand what is happening in figure 3, we can crudely plot the results for $\left\langle r^{2}\right\rangle_{\pi}$ versus their publication date. This is done in figure 4, which shows a time dependence in the calculations with later results agreeing with the measured $\left\langle r^{2}\right\rangle_{\pi}$. More importantly, after selecting those calculations that have accounted in some way for the relevant sources of uncertainty (highlighted in figure 4), we see a consistent picture emerge. All well-controlled lattice calculations agree with 


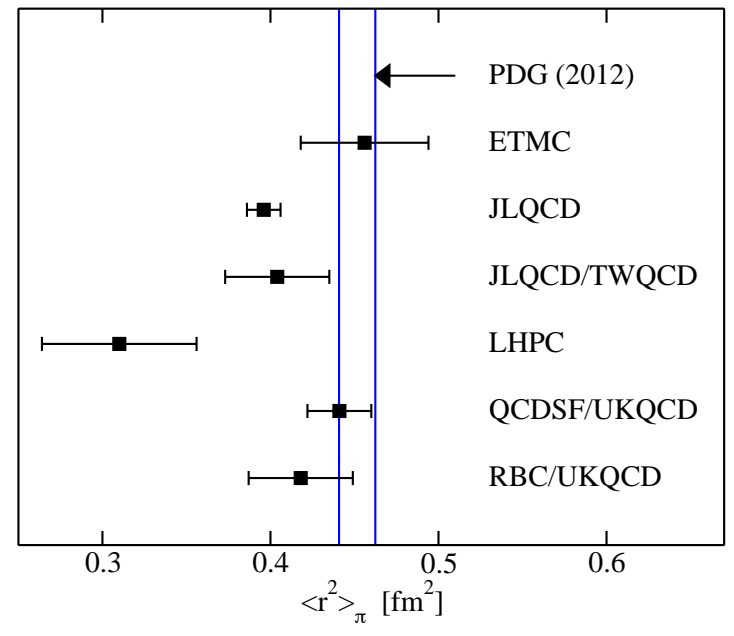

Figure 3: An example of a typical but misleading summary plot. This summary of the pion charge radius $\left\langle r^{2}\right\rangle_{\pi}$, similar to the one in [6], illustrates the dangers in comparing all lattice computations without regard to controlled uncertainties. The values are from PDG [7], ETMC [6], JLQCD [8], JLQCD/TWQCD [9], LHPC [10], QCDSF/UKQCD [11] and RBC/UKQCD [12].

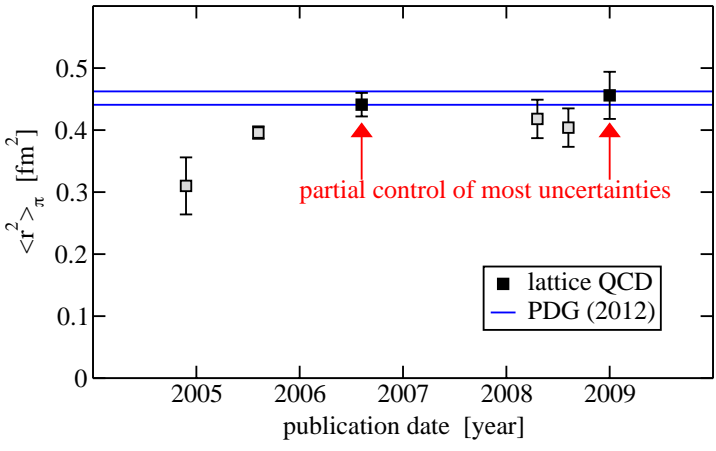

Figure 4: Time history of lattice calculations of the pion charge radius $\left\langle r^{2}\right\rangle_{\pi}$. This time history illustrates that lattice QCD calculations with wellcontrolled uncertainties represent definitive predictions of QCD that can be safely compared to experimental measurements. The impression given by this plot should be contrasted with that given by figure 3, which fails to distinguish those calculations with fully controlled uncertainties. The values are the same as in figure 3 and the two highlighted calculations are, starting with the earliest, by QCDSF/UKQCD [11] and ETMC [6].

each other, as should always happen, and, in this case, agree with the experimental measurement. At this point we are left with only two calculations that meet this burden [6,11], but several of the calculations in figure 4 are ongoing and there are new calculations [13] underway. We should expect in the next few years to have multiple precise and reliable lattice calculations of $\left\langle r^{2}\right\rangle_{\pi}$. As with the results in figures 1 and 2, the easiest observables are calculated first, but this is to be expected from large-scale numerical calculations that face multiple computational thresholds that depend on the quantities of interest. The calculation of $\left\langle r^{2}\right\rangle_{\pi}$ is not only interesting in its own right but also is a good example of how nucleon form factor calculations may progress as the computational barriers are crossed for those observables as well.

Moving beyond the extreme $Q^{2} \rightarrow 0$ limit characterized by the slope of the form factor, lattice calculations are also exploring the non-zero but still low $Q^{2}$ regime of $Q^{2}<1 \mathrm{GeV}^{2}$. Various uncertainties become more challenging with increasing $Q^{2}$, but the low $Q^{2}$ behavior of $F\left(Q^{2}\right)$ can also be calculated with relatively well-controlled uncertainties. This is shown in figure 5 along with the experimental results in the low $Q^{2}$ region. The agreement between lattice QCD and experiment is again rather compelling and bodes well for the eventual determination of nucleon form factors.

\section{Status of nucleon form factors from lattice QCD}

Lattice calculations with nucleons remain a challenge due to the extra computational demands that are required. As such, there is not yet a compelling success story for lattice determinations of nucleon structure, but this needs to be understood in context. It has only been in the last few years that the lattice community has developed sufficient algorithms and garnered enough computing 


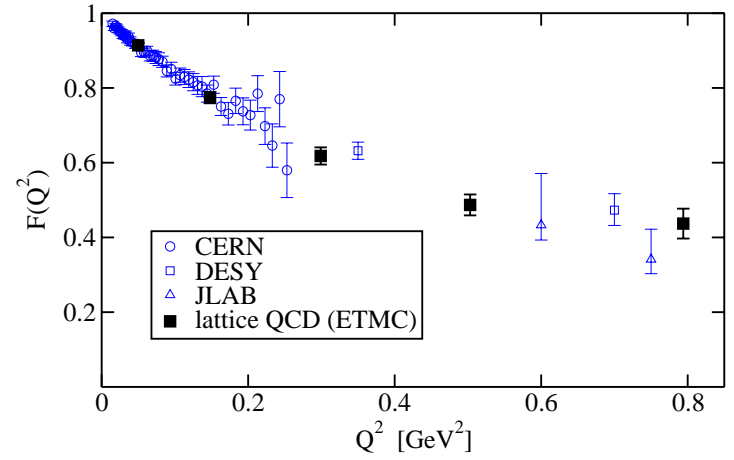

Figure 5: Comparison of the pion form factor $F\left(Q^{2}\right)$ from experiment and lattice QCD. ETMC [6] has performed a lattice calculation with reasonably controlled uncertainties of the low $Q^{2}$ dependence of $F\left(Q^{2}\right)$. This is an example of the level of control sought for the more challenging nucleon form factors. The experimental measurements are from CERN [14], DESY [15, 16] (reanalyzed in [17, 18]) and JLAB [17].

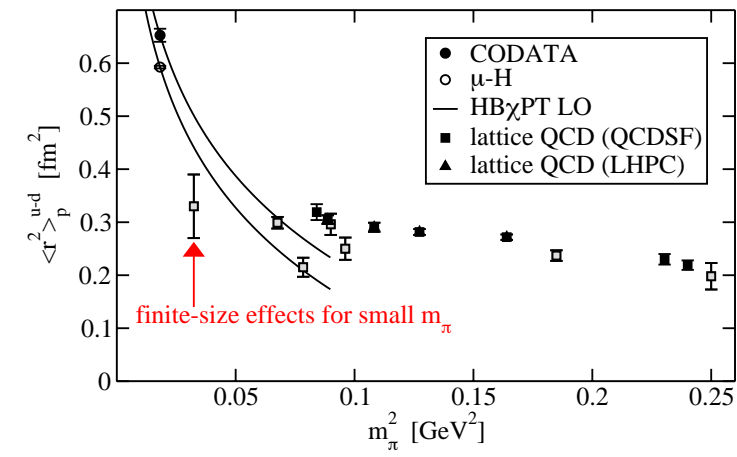

Figure 6: The proton isovector charge radius $\left\langle r^{2}\right\rangle_{p}^{u-d}$. The measured values use CODATA [19] or $\mu-H$ [20] for $\left\langle r^{2}\right\rangle_{P}$ with PDG [7] for $\left\langle r^{2}\right\rangle_{N}$ to form $\left\langle r^{2}\right\rangle_{p}^{u-d}=\left\langle r^{2}\right\rangle_{P}-\left\langle r^{2}\right\rangle_{N}$. These are then matched to chiral perturbation theory [21]. The lattice results are from QCDSF [22] and LHPC [23]. Requiring well-controlled uncertainties likely eliminates the apparent tension between lattice QCD, chiral perturbation theory [21] and the measurements.

power to calculate even $m_{N}$ at the few-percent level. Thus it should not be surprising that current calculations of nucleon structure have produced mixed results, with some encouraging agreements and some noticeable disagreements. As illustrated by the pion form factor, this is how we expect lattice calculations to look before fully controlled uncertainties are achieved.

With this in mind, I simply summarize the current status of nucleon calculations with a few examples. To compare with the discussion of the pion charge radius, I start with the proton charge radius defined similarly,

$$
\left\langle P, p\left|J_{\mu}^{f}\right| P, k\right\rangle=K_{\mu}^{1} F_{1}^{f}\left(Q^{2}\right)+K_{\mu}^{2} F_{2}^{f}\left(Q^{2}\right) \quad\left\langle r^{2}\right\rangle_{p}^{f}=-\left.6 \frac{d F_{1}^{f}\left(Q^{2}\right)}{d Q^{2}}\right|_{Q^{2}=0},
$$

where $K_{\mu}^{i}$ are kinematic functions of $p$ and $k$. From the outset, I have to make a sacrifice and instead focus on the isovector radius $\left\langle r^{2}\right\rangle_{p}^{u-d}=\left\langle r^{2}\right\rangle_{p}^{u}-\left\langle r^{2}\right\rangle_{p}^{d}$. This restriction eliminates so-called disconnected diagrams that are an additional source of uncertainty. Furthermore, I avoid any statements about extrapolations to the physical point and simply examine the lattice results as functions of the artificially heavy pion mass $m_{\pi}$ used in the calculations. These are shown in figure 6. To avoid unnecessary clutter, only two lattice calculations are shown as examples, and only the uncertainties due to numerically integrating the path integral are shown. Considering all results in figure 6 without regard to the remaining uncertainties in the problem, there would be an apparent disagreement between lattice calculations and the measured value. Additionally, there would seem to be a significant disagreement with the expectations of heavy baryon chiral perturbation theory. However, as with the early calculations for the pion form factor, the failure to accurately control all uncertainties in the lattice calculation may very well be the explanation for both of these observations.

A detailed discussion of the uncertainties for $\left\langle r^{2}\right\rangle_{p}^{u-d}$ is beyond the scope of these proceedings, but I will examine one uncertainty that may be a concern: finite-size effects. Lattice calculations 


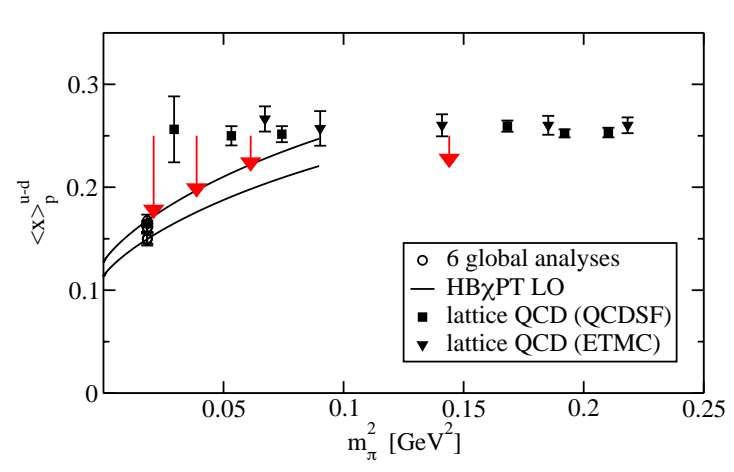

Figure 7: The isovector momentum fraction $\langle x\rangle_{p}^{u-d}$. The lattice results are QCDSF [24] and ETMC [25]. The results from global analyses are collected in [26]. The leading-order results from chiral perturbation theory $[27,28]$ are matched to the largest and smallest results from the global analyses to indicate the impact of the variations among these results. The red arrows indicate the possible impact of the uncertainty, suggested by ETMC [29] and LHPC [30], due to excited-state pollution in the lattice calculations as described in the text.

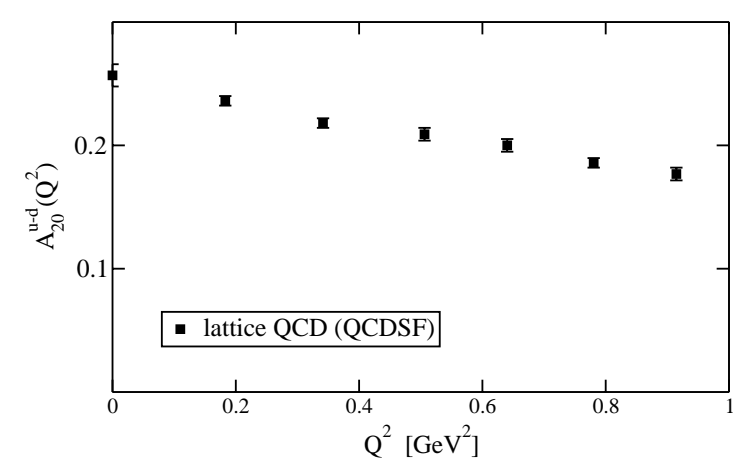

Figure 8: The isovector $A_{20}^{u-d}\left(Q^{2}\right)$ generalized form factor. The moments of the generalizedparton distributions of the proton can be calculated using lattice QCD techniques. The generalized form factor $A_{20}\left(Q^{2}\right)$ shown here is related to the first $x$-moment of the sum of the $H\left(x, \xi, \Delta^{2}\right)$ and $E\left(x, \xi, \Delta^{2}\right)$ GPDs in which $Q^{2}=\Delta^{2}$. The results from [31] are shown as an example. The absence of experimental results emphasizes the prospects for genuine predictions from lattice QCD once all uncertainties are reliably controlled.

are performed at a finite physical volume $L^{3}$ and correspondingly the allowed momentum modes are discretized. Forming the derivative of the form factors requires taking finite differences in $Q^{2}$. Furthermore, this quantity is expected to diverge in the chiral limit, so we may reasonably expect an enhanced sensitivity to the volume, which acts as the infrared regulator for lattice QCD. Due to the demanding nature of nucleon calculations, the large volume limit is difficult to study, especially as $m_{\pi}$ is decreased. Consequently, there is not much evidence for or against a strong finite-size effect for $\left\langle r^{2}\right\rangle_{p}^{u-d}$. In lieu of a full study of the $L$ dependence, we can require the usual rule of thumb $m_{\pi} L>4$. This restriction in figure 6 clearly alleviates the disagreement with the measured value for $\left\langle r^{2}\right\rangle_{p}^{u-d}$ and substantially weakens the tension with the expectations from chiral perturbation theory, suggesting that calculations with larger $L$ may help resolve these puzzles.

Nucleon form factors are challenging enough; turning to moments of parton and generalizedparton distributions increases the difficulties further. In figure 7, I show two example calculations of the average longitudinal-momentum fraction $x$ in the proton $\langle x\rangle_{p}^{u-d}$ given by

$$
\left\langle P, p\left|O_{\mu \nu}^{f}\right| P, k\right\rangle=K_{\mu \nu}^{A} A_{20}^{f}\left(Q^{2}\right)+K_{\mu \nu}^{B} B_{20}^{f}\left(Q^{2}\right)+K_{\mu \nu}^{C} C_{2}^{f}\left(Q^{2}\right) \quad\langle x\rangle_{P}^{f}=\int_{-1}^{1} d x x q^{f}(x)=A_{20}^{f}(0),
$$

where the $K_{\mu \nu}^{i}$ are kinematic functions, $O_{\mu \nu}^{f}=\bar{q}_{f} \gamma_{\{\mu} D_{v\}} q_{f}$ are symmetric traceless twist-two operators, and $q^{f}(x)$ can be related to the quark (anti-quark) parton distribution functions (PDFs) for $x>0(x<0)$. Generalizations to higher moments $\left\langle x^{n}\right\rangle_{P}^{f}$ are possible. The isovector combination is used for the same reasons as discussed regarding $\left\langle r^{2}\right\rangle_{p}^{u-d}$. There is an apparent disagreement with the measured value, but again the issue boils down to fully controlling the relevant uncertainties. An important uncertainty for $\langle x\rangle_{p}^{u-d}$ appears to be a finite-size effect due to excited-state pollution. (This is a technical issue related to having a large enough separation between correlation functions 
in the calculation.) In the case of $\langle x\rangle_{p}^{u-d}$ there are indications from lattice calculations that this effect is occurring. The red arrows in figure 7 show the sort of corrections suggested by direct calculations. These corrections are not universal and can not readily be applied to the results of other computations, so we can only note that the sign of the correction and its the $m_{\pi}$ dependence seems to reduce the disagreement with the measured $\langle x\rangle_{p}^{u-d}$.

I have focused on $\left\langle r^{2}\right\rangle_{p}^{u-d}$ and $\langle x\rangle_{p}^{u-d}$ because they are commonly used as benchmarks for lattice calculations of nucleon structure. Just as the calculation of $m_{N}$ was an essential achievement for making progress, the successful determination of $\left\langle r^{2}\right\rangle_{p}^{u-d},\langle x\rangle_{p}^{u-d}$ and other similar observables is a necessary step towards the broader structure program envisioned by the lattice community. As just one example of that effort, I consider the generalized-parton distributions (GPDs). As for the PDFs, lattice calculations focus on moments in $x$. The first moments of the $H$ and $E$ GPDs are given by the generalized form factors $A_{20}\left(Q^{2}\right), B_{20}\left(Q^{2}\right)$ and $C_{2}\left(Q^{2}\right)$. One example is

$$
\int_{-1}^{1} d x x\left(H^{f}\left(x, \xi, \Delta^{2}\right)+E^{f}\left(x, \xi, \Delta^{2}\right)\right)=A_{20}^{f}\left(\Delta^{2}\right)+B_{20}^{f}\left(\Delta^{2}\right),
$$

where the definitions of the $H$ and $E$ GPDs and further details are available in [32]. There have been several lattice calculations of these form factors, and one recent example is shown in figure 8 . The calculation of $A_{20}^{u-d}\left(Q^{2}\right)$ is an extension of $\langle x\rangle_{p}^{u-d}$ and all issues relevant for $\langle x\rangle_{p}^{u-d}$ are expected to occur for $A_{20}^{u-d}\left(Q^{2}\right)$ as well. In fact, $A_{20}^{u-d}(0)=\langle x\rangle_{p}^{u-d}$. One goal of the long-term lattice effort on nucleon structure is the determination of the low moments of all the nucleon GPDs. The restriction to moments in $x$ is a significant limitation to PDFs, but for GPDs the additional information on the $Q^{2}=\Delta^{2}$ dependence adds valuable information on the GPDs that is complimentary to that which is accessible from experimental measurements. Thus well-controlled calculations of the $Q^{2}$ dependence of form factors like $A_{20}\left(Q^{2}\right)$ will yield genuine predictions from lattice QCD for the nucleon and will open several avenues to rich physics topics including the spin decomposition and transverse structure of the nucleon.

\section{Conclusions}

In the last decade, lattice QCD has shown itself to be capable of precision calculations. The initial successes were understandably limited to those observables for which all uncertainties could be controlled with the least computational resources. However, recent calculations, such as the determination of $m_{N}$, indicate that lattice computations of more demanding hadronic quantities should become feasible in the years to come.

The pion form factor is currently a well-determined quantity from lattice $\mathrm{QCD}$, with further improvements expected. It stands as an example of the way forward for equally well-controlled lattice calculations of nucleon structure. There will be continued emphasis on benchmark observables, such as $\left\langle r^{2}\right\rangle_{p}^{u-d}$ and $\langle x\rangle_{p}^{u-d}$, in order to establish control of all relevant uncertainties for calculations of nucleon matrix elements. This renewed focus on carefully accessing each uncertainty is already shedding some light on current puzzles facing lattice calculations of nucleon structure and will eventually lead to precise and reliable calculations that can safely be compared to experimental measurements. However, the real promise of lattice calculations of nucleon matrix elements ultimately lies in determining observables that lie beyond the reach of measurements yet offer the possibility of insight into the deeper mechanisms behind nucleon structure. 


\section{References}

[1] S. Bethke et al., Workshop on Precision Measurements of $\alpha_{s}$ (2011), arXiv:1110.0016.

[2] J. Laiho, E. Lunghi, and R. S. Van de Water, Phys.Rev. D81, 034503 (2010), arXiv:0910.2928, Updated averages available online at http://www.latticeaverages.org/.

[3] BMW Collaboration, S. Durr et al., Science 322, 1224 (2008), arXiv:0906.3599.

[4] PACS-CS Collaboration, S. Aoki et al., Phys.Rev. D79, 034503 (2009), arXiv:0807.1661.

[5] ETMC Collaboration, C. Alexandrou et al., Phys.Rev. D80, 114503 (2009), arXiv:0910.2419.

[6] ETMC Collaboration, R. Frezzotti et al., Phys.Rev. D79, 074506 (2009), arXiv:0812.4042.

[7] Particle Data Group, J. Beringer et al., Phys.Rev. D86, 010001 (2012).

[8] JLQCD Collaboration, S. Hashimoto et al., PoS LAT2005, 336 (2006), hep-lat/0510085.

[9] JLQCD/TWQCD Collaboration, T. Kaneko et al., PoS LATTICE2008, 158 (2008), arXiv:0810.2590.

[10] LHPC Collaboration, F. D. Bonnet et al., Phys.Rev. D72, 054506 (2005), hep-lat/0411028.

[11] QCDSF/UKQCD Collaboration, D. Brommel et al., Eur.Phys.J. C51, 335 (2007), hep-lat/0608021.

[12] RBC/UKQCD Collaboration, P. Boyle et al., JHEP 0807, 112 (2008), arXiv:0804.3971.

[13] B. B. Brandt, A. Juttner, and H. Wittig, Proceedings of Hadron 2011 (2011), arXiv:1109.0196.

[14] NA7 Collaboration, S. Amendolia et al., Nucl.Phys. B277, 168 (1986).

[15] H. Ackermann et al., Nucl.Phys. B137, 294 (1978).

[16] P. Brauel et al., Z.Phys. C3, 101 (1979).

[17] JLab $F_{\pi}$ Collaboration, V. Tadevosyan et al., Phys.Rev. C75, 055205 (2007), nucl-ex/0607007.

[18] Jefferson Lab, G. Huber et al., Phys.Rev. C78, 045203 (2008), arXiv:0809.3052.

[19] P. J. Mohr, B. N. Taylor, and D. B. Newell, CODATA (2010), arXiv:1203:5425.

[20] R. Pohl et al., Nature 466, 213 (2010).

[21] V. Bernard, N. Kaiser, J. Kambor, and U. G. Meissner, Nucl.Phys. B388, 315 (1992).

[22] QCDSF/UKQCD Collaboration, S. Collins et al., Phys.Rev. D84, 074507 (2011), arXiv:1106.3580.

[23] LHPC Collaboration, S. Syritsyn et al., Phys.Rev. D81, 034507 (2010), arXiv:0907.4194.

[24] Private communication from the QCDSF collaboration.

[25] ETMC Collaboration, C. Alexandrou et al., Phys.Rev. D83, 114513 (2011), arXiv:1104.1600.

[26] D. B. Renner, PoS LAT2009, 018 (2009), arXiv:1002.0925.

[27] D. Arndt and M. J. Savage, Nucl.Phys. A697, 429 (2002), nucl-th/0105045.

[28] J.-W. Chen and X.-D. Ji, Phys.Lett. B523, 107 (2001), hep-ph/0105197.

[29] ETMC Collaboration, S. Dinter et al., Phys.Lett. B704, 89 (2011), arXiv:1108.1076.

[30] J. Green et al., PoS LATTICE2011, 157 (2011), arXiv:1111.0255.

[31] QCDSF Collaboration, A. Sternbeck et al., PoS LATTICE2011, 177 (2011), arXiv:1203.6579.

[32] X.-D. Ji, Phys.Rev.Lett. 78, 610 (1997), hep-ph/9603249. 